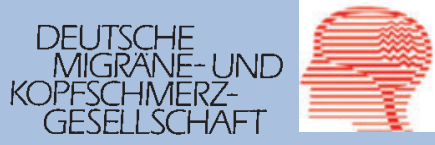

\title{
Entspannung mit dem Smartphone bei Migräne?
}

\author{
*** Minen MT, Adhikari S, Seng EK, Berk T, \\ jinich S, Powers SW, Lipton RB. Smartphone- \\ based migraine behavioral therapy: a single-arm \\ study with assessment of mental health predic- \\ tors. Digital Medicine 2019; 2: 46
}

Da nicht alle Migränepatienten einen guten Zugang zu Fachtherapeuten haben, könnten geeignete Smartphone-Applikationen eine Übergangslösung darstellen.

\section{Zusammenfassung}

In der Migränebehandlung ist die Progressive Muskelrelaxation (PMR) ein leitliniengerechtes verhaltenstherapeutisches Entspannungsverfahren. Da nicht alle Patienten Zugang zu (realen) Kopfschmerztherapeuten und den entsprechenden Behandlungen haben, untersuchten die Autoren, ob eine Smartphone-Applikation für die Anwendung von PMR (RELAXaHEAD App) bei Migränepatienten erfolgreich eingesetzt werden kann.

Hierzu wurden 51 Patienten (48 weiblich) einer neurologischen Universitätspraxis rekrutiert, welche die ICHD-Diagnose Migräne hatten, keine Verhaltenstherapie innerhalb des letzten Jahres erhielten und über ein Smartphone verfügten (willkürliche Stichprobe). Die durchschnittliche Kopfschmerzhäufigkeit betrug 13 Tage pro Monat, $63 \%$ hatten eine schwere Beeinträchtigung (MIDAS-Score). Die Patienten erhielten die Aufgabe, über 90 Tage täglich ein Kopfschmerztagebuch auszufüllen und eine 20 -minütige PMR durchzuführen.

An durchschnittlich ca. 22 Tagen wurde die PMR durchgeführt (Durchschnitt ca. $11 \mathrm{Mi}$ nuten pro Anwendung). Knapp die Hälfte benutzte die PMR mindestens 1-mal pro Woche, knapp ein Drittel mindestens 2-mal pro Woche. Interessanterweise zeigten Patienten mit mehr als 2 PMR-Durchführungen pro Woche im ersten Monat eine Reduktion um durchschnittlich vier Kopfschmerztage im darauffolgenden Monat, die mit weniger als 2 PMR-Durchführungen nur eine Reduktion um durchschnittlich 2 Kopfschmerztage. Dieses Ergebnis ist allerdings angesichts der im Verlauf stark nachlassenden Dokumentation der Kopfschmerzen im Tagebuch mit Vorsicht zu interpretieren. Zu Beginn der Studie erfasste Depressionswerte waren mit einer geringeren Nutzung der App verbunden, Angstwerte mit einer erhöhten. Hier dürften Aspekte von Motivation, Symptomschwere und Erwartungen eine Rolle spielen. Es zeigte sich im Verlauf der Wochen eine Abnahme der Verwendung des Kopfschmerztagebuchs (von $100 \%$ auf $49 \%$ mindestens 1-mal pro Woche) als auch der PMR-Anwendung (von $84 \%$ auf $29 \%$ mindestens 1-mal pro Woche).

\section{Kommentar}

Smartphone-Anwendungen im therapeutischen Setting erfreuen sich großer Beliebtheit. Knapp $90 \%$ der Menschen besitzt ein Smartphone und verfügt so über einen potenziellen und preiswerten Zugang zu solchen Behandlungsmethoden. Die umfassende wissenschaftliche Untersuchung der Effekte hingegen liegt allerdings noch deutlich zurück.

Die Autoren stellen die Grenzen ihrer Studie ausführlich dar, beispielsweise die geringe Stichprobengröße, keine randomisierte Auswahl und dass es für zukünftige Studien nicht nur einen Behandlungsarm geben sollte. Viele weitere beeinflussenden Faktoren wurden nicht bzw. konnten nicht kontrolliert werden, beispielsweise was Patienten nebenher am Smartphone taten oder ob sie die Dosierung ihrer Medikamente veränderten. Auch ist die PMR in der realen Verhaltenstherapie nur ein Baustein. Die reduzierte Adhärenz im Verlauf der Zeit lässt sich allerdings auch bei Behandlungen ohne Smartphone beobachten. Ein Pluspunkt der Studie ist, dass auch schwer beeinträchtige Patienten eingeschlossen wurden, was viele Studien im Rekrutierungsprozess nicht machen, sondern vielmehr nach motivierten Patienten suchen.

Es bleibt zu testen, inwiefern reale Verhaltenstherapien durch Smartphone-Anwendungen ergänzt werden können. So könnten sowohl Patienten, als auch Therapeuten in ihrer Arbeit unterstützt und entlastet werden. Insbesondere motivationale Aspekte bzw. begleitende Komorbidität könnten dann von dem Therapeuten mehr berücksichtigt werden und so die Adhärenz erhöhen. Zumindest als Übergangslösung bei langen Wartezeiten auf Therapieplätze könnten Smartphone-Anwendungen zukünftig eine größere Rolle spielen. Allerdings bedarf es dazu viel mehr Forschung. Es müssen Qualitätsstandards geschaffen werden, die analog zu Therapieleitlinien angewendet werden, um Patienten und Therapeuten wirksame Smartphone-Anwendungen empfehlen zu können. Medizinische Smartphone-Anwendungen müssen etabliert werden, die für ihre Zulassung entsprechende Kriterien erfüllen.

Die Forschung in diesem Bereich ist wichtig, um therapeutisch wirksame und sichere Smartphone-Anwendungen zu ermöglichen. Es werden noch zu viele Apps angeboten, die nicht auf wissenschaftlichen Erkenntnissen basieren. Kunden (respektive Patienten und Therapeuten) müssen wissen, dass die Verfügbarkeit einer App per se weder Effektivität, Sicherheit, noch die Einhaltung bestimmter Qualitätsstandards durch die Entwickler garantiert.

Thomas Dresler, Tübingen 


\section{Calcitonin gene-related peptide (CGRP) als Marker für die Krankheitsaktivität bei Clusterkopfschmerz?}

**(*) Snoer A, Vollesen ALH, Beske RP, Guo S, Hoffmann J, Fahrenkrug J, Jorgensen NR, Martinussen T, Jensen RH, Ashina M. Calcitoningene related peptide and disease activity in cluster headache. Cephalalgia 2019: 39(5) 575-584

Trotz recht hoher Patientenzahl und aufwendigem, placebokontrolliertem Studiendesign bleiben die Daten inkonklusiv.

\section{Hintergrund}

CGRP spielt eine wichtige Rolle bei Clusterkopfschmerz-Attacken [1], was insbesondere aufgrund von Hinweisen für die Wirksamkeit von CGRP-Antikörpern in der Therapie vom episodischen Clusterkopfschmerz interessant ist. Aber auch andere Neuropeptide könnten in der Pathophysiologie des Clusterkopfschmerz eine Rolle spielen.

\section{Zusammenfassung}

In der vorliegenden, randomisierten, doppelblinden, placebokontrollierten Crossover Studie wurden die Neuropeptide CGRP, Pituitary adenylatecyclase activating polypeptide (PACAP) und vasoaktives intestinales Peptid (VIP) in episodischen (eCKS) und chronischen (cCKS) Clusterkopfschmerz-Patienten vor und während experimentellen Clusterkopfschmerz-Attacken (induziert durch intravenöse (GRP-Gabe) untersucht.

Es nahmen 31 Patienten teil ( 9 eCKS in aktiver Phase, 9 eCKS in Remission und 13 cCKS). Vor (Baseline) und nach Infusion von $30 \mu \mathrm{g}$ CGRP bzw. Placebo an 2 Untersuchungstagen erfolgten cubitale Blutentnahmen zu mehreren Zeitpunkten. Sofern eine Clusterkopfschmerz-Attacke auftrat, erfolgten weitere Blutentnahmen zu definierten Zeitpunkten. Die Bestimmung der Neuropeptide erfolgte mittels Radioimmunoassays.

In 16 aus 31 Patienten konnte eine Clusterkopfschmerz-Attacke induziert werden (keine davon in eCKS-Patienten in Remission) und in 11 aus 16 Attacken konnte eine Blutentnahme stattfinden. In den restlichen 5 Attacken sei die Blutentnahme aufgrund der schnellen Verbesserung der Symptomatik nicht durchführbar gewesen.
In der vorliegenden Arbeit werden Gruppenunterschiede zum Baseline-Zeitpunkt sowie nach CGRP-Infusion abhängig oder unabhängig vom Auftreten einer Clusterkopfschmerz-Attacke berichtet. Der Verlauf über die induzierten Clusterkopfschmerzattacken wird nicht berichtet. Es fanden sich signifikant höhere CGRP-Plasmalevel in eCKS-Patienten in Remission verglichen mit cCKS-Patienten $(100,6 \pm 36,3 \mathrm{pmol} / \mathrm{I}$ vs. $65,9 \pm 30,5 \mathrm{pmol} / \mathrm{l}, \mathrm{p}=0,011)$, aber kein signifikanter Unterschied zu eCKS-Patienten in aktiver Phase $(89,7 \pm 26.9 \mathrm{pmol} / \mathrm{I})$. Nach CGRP-Infusion (aber nicht nach Placebo) kam es zu einem signifikanten Anstieg der CGRP-Spiegel im Vergleich zu den Baseline-Werten $(574,3 \pm 296,4 \mathrm{pmol} / \mathrm{I}$ vs. $81,7 \pm 33,4 \mathrm{pmol} / \mathrm{l} ; \mathrm{p}<0,0001)$, ohne Unterschied zwischen Patienten mit und ohne Attacken.

PACAP38-Serumspiegel waren signifikant höher bei eCKS-Patienten in aktiver Phase im Vergleich zu cCKS-Patienten $(4,0 \pm 0,8 \mathrm{pmol} / \mathrm{l}$ vs. $3,3 \pm 0,7 \mathrm{pmol} / \mathrm{l}$; $\mathrm{p}=0,033)$. Die CGRP-Infusion führte zu keiner Veränderung in den PACAP38-Werten $(p=0,66)$, unabhängig von der Induktion einer Attacke. VIP-Serumspiegel waren zu Baseline nicht signifikant unterschiedlich zwischen den Gruppen, allerdings zeigte sich nach CGRP-Infusion eine signifikante Erhöhung der VIP-Werte im Vergleich zu den Baseline-Werten $(p<0,001)$, nicht jedoch nach Placebo-Gabe. Bei induzierten Attacken kam es dagegen zu einer Reduktion von VIP.

Die Autoren interpretieren die niedrigeren Baseline-CGRP-Spiegel bei cCKS im Vergleich zu eCKS (in Remission) als Hinweis auf eine Depletion von CGRP bei häufigen Clusterkopfschmerz-Attacken. Sie spekulieren auch über einen Zusammenhang mit der (bisher nur aus Pressemitteilungen bekannten) fehlenden Wirkung von CGRP-Antikörpern bei cCKS im Vergleich zu eCKS.

\section{Kommentar}

Der Nachweis relevanter Peptide, insbesondere von CGRP, in primären Kopfschmerzpatienten im peripheren Blut wird aufgrund widersprüchlicher Studienergebnisse kon- trovers diskutiert. In dieser Hinsicht ist das vorliegende Studiendesign mit Nachweis von CGRP, PACAP38 und VIP im Verlauf einer experimentell induzierten Clusterkopfschmerz-Attacke sehr interessant. Es ergeben sich allerdings einige grundsätzliche Einschränkungen: Zwar scheint die Patientenzahl mit 31 Clusterkopfschmerz-Patienten zunächst hoch, es sollte aber bedacht werden, dass nur 11 Clusterkopfschmerz-Attacken ausgewertet werden konnten, die für die Interpretation der Daten in Untergruppen eingeteilt wurden. Zum anderen stellt sich die Frage, wie v. a. der Nachweis von CGRP nach Induktion einer Attacke mittels CGRP interpretiert werden kann. Leider werden die Neuropeptid-Verlaufswerte über die Attacke hinweg im Paper selbst nicht veröffentlicht (können aber nachgefragt werden). Dies wäre gerade für den Verlauf von CGRP nach der Infusion interessant und aufschlussreich gewesen.

In der vorliegenden Studie wurden aktive eCKS-Patienten und cCKS-Patienten eingeschlossen, sofern in den letzten 3 bzw. $8 \mathrm{~h}$ keine Clusterkopfschmerz-Attacke aufgetreten war. Allerdings wurde die letzte Einnahme von Triptanen nicht dokumentiert, obwohl aus zahlreichen früheren Studien bekannt ist, dass die erfolgreiche Behandlung einer Kopfschmerzattacke zu einer signifikanten Reduktion der Blutspiegel auf Baseline-Niveau führt, sodass dies bspw. eine alternative Erklärung für die niedrigen CGRP-Baseline-Spiegel in cCKS (und aktiven eCKS)-Patienten sein könnte [1]. Weiterhin sollte auch die Einnahme einer Prophylaxe als konkurrierende Ursache in Betracht gezogen werden, da aus einer anderen Studie bekannt ist, dass auch die Einnahme einer Prophylaxe zu einer Reduktion der CGRP-Spiegel führen kann [2]. In der vorliegenden Studie nahm ein Teil der Patienten in allen drei Gruppen eine Prophylaxe ein. PACAP wurde bereits in einer kleinen explorativen Studie in Clusterkopfschmerz-Patienten während spontaner Kopfschmerz-Attacken und in Remission untersucht, hier zeigten sich iktal erhöhte PACAP38-Spiegel [3].

Zusammenfassend liefert die Studie interessante Ergebnisse, die aufgrund der Ein- 
schränkungen allerdings mit Vorsicht interpretiert werden sollten und die die Fallstricke der klinischen Forschung in Bezug auf Neuropeptide deutlich werden lässt. Die erneute Durchführung in einer größeren Studienpopulation mit entsprechenden Untergruppen (z. B. Einnahme einer Akutmedikation, Einnahme einer Prophylaxe) wäre sicherlich ein Gewinn.

\section{Katharina Kamm, München}

[1] Edvinsson L, Goadsby P. Neuropeptides in migraine and cluster headache. Cephalalgia 1994; 14: 320

[2] Neeb L, Anders L, Euskirchen P et al. Corticosteroids alter CGRP and melatonin release in cluster headache episodes. Cephalalgia 2015; 35 (4): 317-26

[3] Tuka B, Szabó N, Tóth E et al. Release of PACAP-38 in episodic cluster headache patients - an exploratory study. The journal of headache and pain 2016; 17 (1): 69

\section{INFORMATION}

***** Exzellente Arbeit, die bahnbrechende Neuerungen beinhaltet oder eine ausgezeichnete Übersicht bietet

**** Gute experimentelle oder klinische Studie

*** Gute Studie mit allerdings etwas geringerem Innovationscharakter

** Studie von geringerem klinischen oder experimentellen Interesse und leichteren methodischen Mängeln

* $\quad$ Studie oder Übersicht mit deutlichen methodischen oder inhaltlichen Mängeln

Die Kopfschmerz-News werden betreut von: Priv.-Doz. Dr. Ruth Ruscheweyh, Klinik und Poliklinik für Neurologie, Klinikum der Universität München, Marchioninistr. 15, 81377 München, Tel. 089/440073907, ruth. ruscheweyh@med.uni-muenchen.de

Die Besprechungen und Bewertungen der Artikel stellen die Einschätzung des jeweiligen Autors dar, nicht eine offizielle Bewertung durch die Deutsche Migräne- und Kopfschmerzgesellschaft. 